\title{
Similar effects of mouse and human pub gene on proliferation and embryoid bodies formation in mouse embryonic stem cells in vitro
}

\author{
Ekaterina Novosadova, Nella Khaydarova, Ekaterina Manuilova, Elena Arsenyeva, \\ Andrey Lebedev, Vyacheslav Tarantul, Igor Grivennikov
}

Institute of Molecular Genetics, Russian Academy of Sciences, Moscow, Russia; novek-img@mail.ru, grivigan@mail.ru

Received 18 January 2012; revised 21 February 2012; accepted 27 March 2012

\begin{abstract}
Previously we have demonstrated that pub gene modulates expression of PU.1 transcription factor and plays an important role in differentiation of embryonic stem cells not only at early stages (during formation of embryoid bodies), but also during differentiation into ectodermal, mesodermal and endodermal derivatives in vitro. We have compared the influence of elevated expression of two homological genes mpub and hpub of mouse and human respectively on early stages of differentiation of murine embryonic stem cells. Overexpression of both genes caused an increase in the number of formed embryoid bodies but did not alter the proliferative activity of transfected embryonic stem cells. It was also observed that expression of mpub and hpub led to decreased expression of pu.1 mRNA.
\end{abstract}

Keywords: Embryonic Stem Cells; Transfection; Gene Pub; Proliferation

\section{INTRODUCTION}

Pub belongs to TRIM protein family. This family usually is composed of three zinc-binding domains: RING (R) domain, B-box type 1 (B1) domain and B-box type 2 (B2) domain, followed by coiled-coil (CC) domain [1].

Genes, related to TRIM family are involved in diverse cellular processes, such as growth, differentiation and transcription regulation [2]. For example, the gene coding RFP (RET finger protein) was cloned as transforming gene from human lymphoma cells. Rfp gained its transforming activity only if expressed as recombinant fuse protein when its DNA sequence was integrated with fragment coding tyrosine kinase of RET protein [2]. It was shown that RFP protein is present in nuclei and cytosol of cells of wide range of tissues as well as was shown it's interaction with transcriptional regulator (EPC), these facts suggest possible involvement of the abovementioned TRIM family member in processes of cellular and differentiation and proliferation [2].

Another distinguished member of the current family is the PML protein. PML plays an important role in maintenance of genome stability $[3,4]$.

Mutations and structural changes in domains lead to diseases such as familial Mediterranean fever (FMF), Opitz syndrome, dwarfism, as well as malignancies such as promyelocytic leukemia or thyroid carcinoma.

This suggests that TRIM family members play important role in many biological processes [5].

Previously we have shown that human gene hpub is excessively expressed in human immunoblast lymphomas [6]. In mouse the high level of mRNA of the homologue mpub was observed in spleen, thymus, liver and low level in brain, kidney and skeletal muscle [1]. It was shown that mpub binds the master transcription factor PU.1 and is capable to modulate its transcriptional activity [1].

On the basis of this data and considering Pub structure it can be assumed that current protein possesses regulatory fuctions and is able to influence the processes of cellular growth and differentiation $[7,8]$.

Embryonic stem (ES) cells present a unique model for investigation of processes of differentiation into derivatives of three primary germ layers in vitro as well as in vivo.

In specific cultivation conditions ES cells are capable to go into spontaneous differentiation generating muscular, hematopoietic, neuronal or other derivatives $[9,10]$.

Its also well known that directed differentiation of ES cells can be achieved by addition of different exogenic growth and differentiation factors into the culture media or by the genetic modifications $[11,12]$.

\section{MATERIALS AND METHODS}

\subsection{Cell Cultures}

Mouse R1 line ES cells (kindly presented by Alan 
Nagy, Mount Sinai Hospital, Toronto, Canada) was isolated from agouti-color $(129 / \mathrm{Sv} \times 129 / \mathrm{SvJ}) \mathrm{F} 1$ line mouse blastocytes. Cell were cultured at $37^{\circ} \mathrm{C}$ and with $5 \% \mathrm{CO}_{2}$ in $\alpha$-MEM medium (Sigma, USA) containing $15 \%$ fetal bovine serum (FBS) (Gibco, USA), $0.1 \mathrm{mM}$ 2-mercaptethanol, $2 \mathrm{mM}$ L-glutamin, nonessential amino acids (Gibco, USA), nucleosides, vitamins, and antibiotic gentamicin $(20 \mu \mathrm{g} / \mathrm{ml})$. Mitomycin-C $(3 \mu \mathrm{g} / \mathrm{ml})$ treated primary fibroblasts derived from 11 - 12 day mouse embryos were used as feeder layers. Fibroblast cultures were grown in DMEM (Sigma, USA), containing 10\% FBS, $2 \mathrm{mM}$ L-glutamine and antibiotic gentamycin (20 $\mu \mathrm{g} / \mathrm{ml})$. When ES cells were cultured in absence of feeder layer recombinant leukemia inhibitory factor (LIF) was added into the culture media (Sigma, USA) to final concentration of $10 \mathrm{ng} / \mathrm{ml}$, to block the spontaneous differentiation of ES cells. Cells were passaged every 72 hours.

\subsection{Plasmids Used for ES Cell Transfection}

The $p P B$ plasmid used in current work was constructed on the base of commercially-available $p c D N A 3$ vector (Promega, USA). The recombinant plasmid $p P B$ was obtained by cloning full 4450 b.p. cDNA sequence of human hpub (Blast 2 database number: gi 15208662) between restriction sites HindIII and NotI.

The plasmid pub-myc was kindly provided by kindly provided by Hirofumi Nishizumi, (University of Tokyo, 2-11-16 Yayoi, Bunkyo-ku, Tokyo 113-0032, Japan) [2]. The vector contained cDNA sequence of mouse $p u b$ gene, expressed as fuse protein with myc-epitiope, which allowed further detection of pub protein product, using commercial antibodies against myc-epitope. To obtain the control plasmid k-myc, the pub-myc vector was enzymatically digested by specific endonuclease removing the sequence of $p u b$ between XhoI restriction sites and ligating the sticky ends. Plasmid DNA was isolated from transformed $E$. coli XL1 cells Qiagen DNA isolation kit (USA). Plasmid DNA used for transfection was redissolved in sterile water after ethanol precipitation to final concentration of $2 \mu \mathrm{g} / \mathrm{ml}$.

\subsection{Transfection of ES Cells with Unifectin-56}

The day before transfection ES cells were seeded on $35 \mathrm{~mm}$ Petri dishes covered with gelatin by $5 \times 10^{5}$ per dish with standard culture media. The next day the plasmid DNA was introduced to cells combined with Unifectin-56 (Unifect. The transfection group. Russia) according to producer's protocol. Plasmids k-myc and pub-myc were used for transfection in concentration of 6 mcg per 1 million of cells. For estimation of transfection efficiency the pEGFP-C1 plasmid was used, which con- tained the enhanced green fluorescent protein gene sequence.

\subsection{Isolation of Protein Homogenates from Mammalian Cells}

Protein homogenates were isolated from transfected and control cells and EB as previously reported by as [13].

\subsection{Protein Electrophoresis in Polyacrylamide Gel}

Electrophoretic separation was performed according to Laemmli [14].

\subsection{Western Blotting}

After electrophoretic separation, proteins were transferred on a Hybond ECL nitrocellulose membrane (Amersham, England) using a Bio-Rad blotting unit at 0.8 $\mathrm{mA} / \mathrm{cm}^{2}$ for $90 \mathrm{~min}$. Polyclonal rabbit antibodies against TRIM 14 (Aviva Systems biology, USA) and mouse antibidies anti-myc (Invitrogen, USA) were used for detection of protein bands on the nitrocellulose membrane. Antibodies against rabbit IgG and mouse IgG conjugated with horseradish peroxidase (IMTEKRussia) were used as secondary antibodies. Complexes were visualized using ECL kit (Amersham, England).

\subsection{Test on Pluripotency of Transfected ES Cells}

Alkaline phosphatase and SSEA-1 detection was demonstrated as described [15].

\subsection{Total RNA Isolation and RT-PCR}

Total RNA from ES cells cultivated without the feeder layer in the presence of LIF, EBs was isolated by phenol-chloroform extraction method using a YellowSolve kit (Clonogen, Russia). Reverse transcription was performed using a kit from Silex (Russia) according to the protocol and recommendations of the manufacturer. DNA synthesis was run with $0.5 \mu \mathrm{g}$ total RNA at $37^{\circ} \mathrm{C}$ for $1 \mathrm{~h}$ in $20 \mu \mathrm{l}$ of reaction mixture containing $0.05 \mu \mathrm{g}$ of random hexa-primers and 100 units of moloney murine leukemia virus reverse transcriptase. The reaction was stopped by $10 \mathrm{~min}$ incubation at $70^{\circ} \mathrm{C}$, the cDNA samples were stored at $-20^{\circ} \mathrm{C}$.

Polymerase chain reaction (PCR) was run in $25 \mu \mathrm{lre}$ action mixture containing Taq-buffer, $1.5 \mathrm{mM}$ mixture of dNTP, 1.25 unit "colored" Taq polymerase (Sintol, Russia), $0.5 \mu \mathrm{l}$ cDNA sample and $10 \mathrm{pmol}$ of each of the following primers:

For gapdh: 


\section{5'-TCCATGACAACTTTGGCATTGTGG-3' \\ 5'-GTTGCTGTTGAAGTCGCAGGAGAC-3' \\ For pu. 1 \\ 5'-CCCGGATGTGCTTCCCTTAT-3' \\ 5'-TCCAAGCCATCAGCTTCTCC-3' \\ For mpub: \\ 5'-CCCATTTGGAAGACGCCG-3' \\ 5'-AGGGTGGCTCAGCTCCG-3' \\ PCR conditions for the gene gapdh were the following:} start, 2 min, $95^{\circ} \mathrm{C}, 27$ cycles: $95^{\circ} \mathrm{C}, 1 \mathrm{~min} ; 66^{\circ} \mathrm{C}, 45 \mathrm{~s}$; $72^{\circ} \mathrm{C}, 45 \mathrm{~s}$. PCR conditions for the gene pu. 1 were the following: start, $2 \mathrm{~min}, 52^{\circ} \mathrm{C} ; 35$ cycles: $94^{\circ} \mathrm{C}, 30 \mathrm{~s} ; 59^{\circ} \mathrm{C}$, $30 \mathrm{~s} ; 72^{\circ} \mathrm{C}, 30 \mathrm{~s}$.

The PCR products were separated in $1.5 \%$ agarose gel, visualized using ethidium bromide, and analyzed in BioDocAnalyze system (Biometra, FRG).

Fluorescence intensity was measured for the following products: 375 bp (gapdh), 120 bp (pu.1), 328 bp (mpub) in UV light.

\subsection{Evaluation of ES Cells Proliferation}

To evaluate cell growth, ES cells were plated on the dishes $(d=35 \mathrm{~mm})$ about 150,000 cells per dish, covered with gelatin, in $2 \mathrm{ml}$ standard medium for stem cells with addition of LIF $(10 \mathrm{ng} / \mathrm{ml})$. Cell numbers were estimated on day 3 after plating by direct counting in Goryaev chamber under Olympus CKX41 microscope (Olympus, Japan). Not less than six dishes were counted for each line.

\subsection{Induction of ES Cells Differentiation and EB Formation}

To induce differentiation with formation of EB, the cells were isolated form the feeder layer fibroblasts: treated with tripsin, centrifuged, and then the suspension was incubated in a Petri dish $(\mathrm{d}=60 \mathrm{~mm})$ (Nunc, Denmark) for 15 - $30 \mathrm{~min}$. During this time, the main part of the fibroblasts attached to the bottom of the dish, while stem cells remained in suspension. For EB formation, suspended cells were plated on Petri dish $(d=35 \mathrm{~mm})$ (Nunc, Denmark), about 200,000 cells per dish, or on 96-well U-form plates (Costar, Netherlands), 1000 cells per well and then placed into $\mathrm{CO}_{2}$-incubator $\left(5 \% \mathrm{CO}_{2}\right)$. The EB were counted on day 3 after formation of the first EB.

\subsection{Statistical Analysis}

The results were processed with SigmaPlot for Windows and Origin for Windows (Jandel Scientific). The results were presented as mean $\pm \mathrm{SE}$. The sets of data were compared using the multiway ANOVA. Difference was considered significant at $\mathrm{p}<0.05$.

\section{RESULTS}

For obtaining the tranisntly-transfected polyclonal lines of ES cells we have used the pub-myc plasmid, bearing cDNA sequence of murine mpub coexpressed with myc epitope. The above plasmid was kindly provided by our Japanese colleagues [1]. k-myc vector was constructed based on the pub-myc plasmid in which the pub sequence was removed by endonuclease digestion and was used as the control.

By transfection of ES cell line R1 we have obtained two polyclonal cultures of ES-pub-myc (carrying transgene) and ES-myc control line. By using RT-PCR we have shown the increase of expression of mpub gene both in $m p u b$ transfected undifferentiated. ES cells (ESpub-myc) and in embryoid bodies formed by these cells, compared to control line ES-myc (data not shown).

Earlier we have obtained the polyclonal line ES-hpub (carrying human $h p u b$ gene) and corresponding control line ES-DNA3, as well as embryoid bodies from these lines [16].

Using Western-blot analysis we have shown the presence of protein product hPUB in hpub transfected cells and embryoid bodies formed by them (Figure 1).

Also the presence of protein product mPub was shown in cell line ES-pub-myc (Figure 2).

Next we have estimated the level of endogenous expression of $m p u b$ in polyclonal lines obtained earlier, carrying the human hpub gene. As a consequence of earlier experiments it was shown that in cells carrying human transgenic hpub (ES-hpub) as well as in control line the expression of endogenous mpub was unaltered (Figure 3).

All transfected ES cells expressed cell surface markers specific for undifferentiated ES cells including stagespecific embryonic antigen SSEA-1 and alkaline phosphatase (Figure 4).

It was shown previously that mpub gene product sup-

$48 \mathrm{kD}$

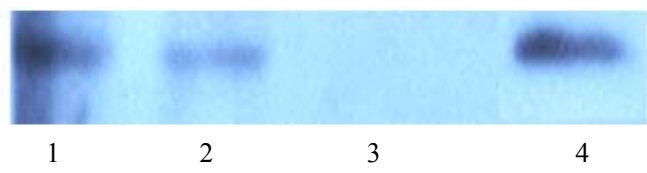

Figure 1. Western blotting of hpub-transfected undifferentiated ES cells end EB. 1. ES-hPub; 2. EB-hPub; 3. ESDNA3 (control); 4. EB-DNA3 (control); 5. The positive control Jurcat cell.

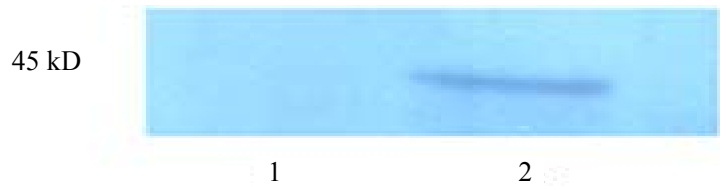

Figure 2. Western blotting of $m p u b$-transfected undifferentiated ES cells. 1. ES-myc (control); 2. ES-pub-myc. 


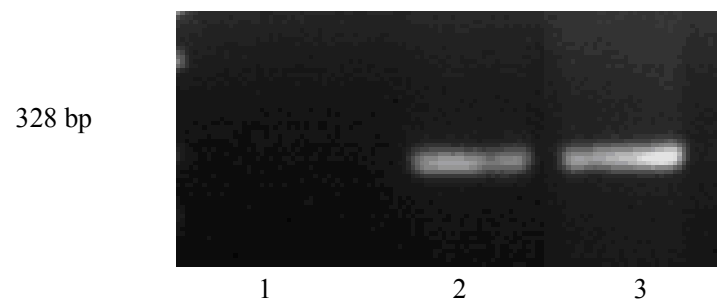

Figure 3. Expression of endogenous mpub in undifferentiated ES cells transfected with human hpub. 1. Negative control (mixture of RNA for RT-PCR); 2. ES-DNA3 cells; 3 . ES-hPub cells.

(a)

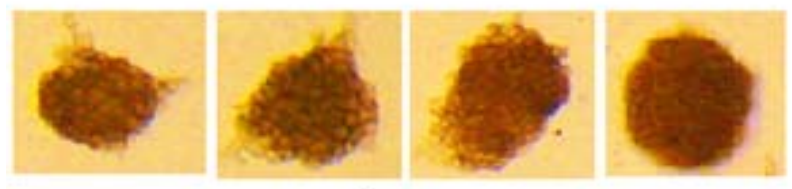

(b)

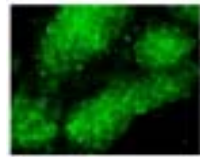

1

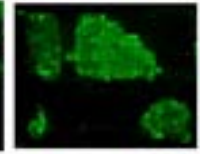

2

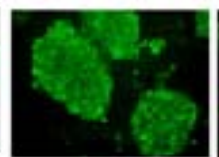

3

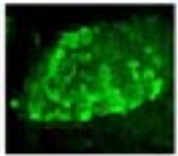

4
Figure 4. Expression of cell surface markers by transfected MESC. (a) Alkaline phosphatase, (b) SSEA-1. 1. Cells ES-myc (control); 2. Cells ES-pub-myc; 3. Cells ES- DNA3 (control); 4. Cells ES-hpub, $\times 200$.

presses the biological activity of transcriptional factor PU.1 [1]. However, the data concerning the possible influence of mpub on $p u .1$ expression level are absent in the current literature.

We have examined how the overexpression of $m p u b$ and $h p u b$ would influence the expression of endogenous $p u .1$. We have shown that overexpression of either human $h p u b$ or murine mpub caused decrease in expression of endogenous pu.1 in cultured transfected ES cells (Figure 5).

Comparing the proliferative activity of all four experimental and control lines, no difference was revealed (Figure 6).

The above data suggests $m p u b$ gene product as well as hpub do not have considerable influence on proliferaition of undifferentiated ES cells. Next we have compared the time and the number of formed embryoid bodies in cells overexpressing mpub and hpub and in control lines.

It was shown that the ES-hpub cell line expressing $h p u b$ gene, as well as in the line ES-pub-myc overexpressing murine mpub the embryoid bodies formed approximately 1 day earlier than in control lines (transfected with pcDNA3 and k-myc plasimds resprectively).

Data, presented on Figure 7, gives evidence that expression of $m p u b$ or hpub in ES cells substantially influences the early stages of their differentiation, namely the (a)

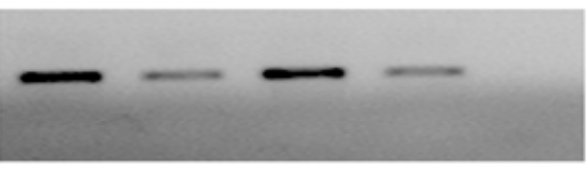

(b)

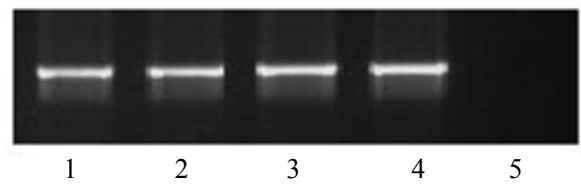

Figure 5. Expression of pu.1. (a) and gapdh (b) in undifferentiated ES cells transfected with human and mouse genes $p u b$. 1. Cells ES-myc (control); 2. Cells ES-pubmyc; 3. Cells ES - DNA3 (control); 4. Cells ES-hpub; 5. Negative control (mixture of RNA for RT-PCR).

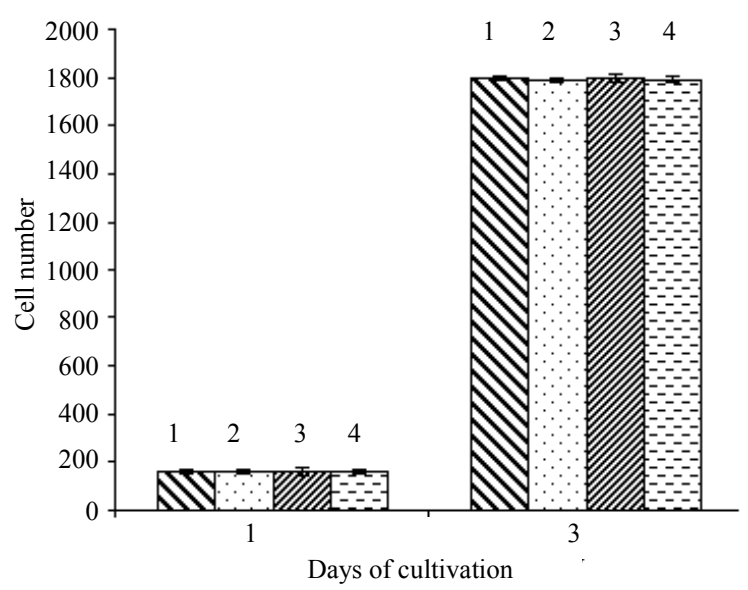

Figure 6. Influence of overexpression of $h p u b$ and $m p u b$ genes on proliferative activity of ES cells. 1. ES-DNA3 (control); 2. ES-hPub; 3. ES-myc (control); 4. ES-pubmyc.

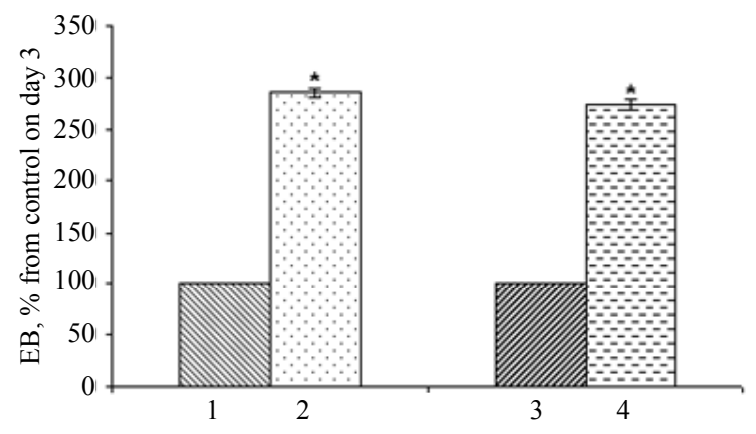

Figure 7. Influence of overexpression of $h p u b$ and $m p u b$ genes on EB formation. 1. EB-DNA3 (control); 2. EB-hPub; 3. EB-myc (control); 4. EB-pub-myc. Maximal amount of EBs in control line at 1 and 3 days after the initiation of formation was taken up as $100 \%$. ${ }^{*} \mathrm{p}<0.05$.

embryoid body formation. After three days after seeding in conditions inducing the formation of EBs their number in ES-hPub and ES-pub-myc cultures was approximately 
2.5 times above the number in control cultures.

\section{DISCUSSION}

It has been established that $p u b$ gene can modulate the transcriptional activity of PU.1, whisch is the one of the most significant factors controlling hematopoietic differentiation. [1] Recently it was shown, that PU.1 participates in the maintenance of pluripotence of ES cells and demonstrates its effects on earliest stages of differentiation of these cells [17].

The goal of our research is studying the $p u b$ gene functions. For this purpose we have created earlier the experimental models of murine ES cells overexpressing human hpub gene and with decreased expression of endogenous mpub of mouse. We have shown significant effect of this gene on various differentiation stages and directions of cellular differentiation, including the EB formation [18].

It is unquestionable that correct transgene function depends not only on its expression stability but also its possible transcriptional and posttranscriptional modifications, such as processing, protein translation regulation to allow the correct further interactions with proteins and other molecules. This is especially actual when heterologous expression systems are used in the experiment. Due to a high homology level of human hpub and murine mpub genes, we have made an assumption that murine cells transfected with human hpub sequence are capable to synthesize the mature biologically active protein Pub.

To check our assumption we have compared the effects of genes hpub and mpub in populations of transfected ES cells. Comparing the proliferative activity of experimental and control lines no significant difference between these lines were observed. The data on time of EB formation and number of formed embryoid bodies did not reveal any differences in effects of mpub and hpub.

As we have previously demonstrated [1] pub gene product physically interacts with PU.1 transcription factor, which is the one of the most important proteins in regulation of hematopoiesis, inhibiting its activity. We were the first to demonstrate that either $m p u b$ or $h p u b$ overexpression led to decrease in expression of $p u .1$ mRNA in mouse ES cells.

In summary, as result of carried experiments we have shown the analogous influence of overexpression of $m p u b$ and hpub genes on proliferation and embryoid body formation in cultured murine ES cells, as well as its negative influence on $p u .1$ expression level.

\section{ACKNOWLEDGEMENTS}

This study was partially supported by Russian Foundation for Basic Research (Grant N 11-04-01337, 10-04-00656a) and Ministry of educa- tion and science of Russian Federation (Grant N 14.740.11.0171)

\section{REFERENCES}

[1] Hirose, S., Nishizumi, H. and Sakano, H. (2003) Pub, a novel PU.1 binding protein, regulates the transcriptional activeity of PU.1. Biochemical and Biophysical Research Communications, 311, 351-360. doi:10.1016/i.bbrc.2003.09.212

[2] Shimono, Y., Murakami, H., Hasegawa, Y. and Takahashi, M. (2000) RET finger protein is atranscriptional repressor and interacts with enhancer of polycomb that has dual transcriptional functions. The Journal of Biological Chemistry, 275, 39411-39419. doi:10.1074/jbc.M006585200

[3] Jensen, K., Shiels, C. and Freemont, P. (2001) PML protein isoforms and the RBCC/TRIM motif. Oncogene, 20, 7223-7233. doi: $10.1038 /$ sj.onc. 1204765

[4] Zhong, S., Hu, P., Y,e T., Stan, R., Ellis, N. and Pandolfi, P. (1999) A role for PML and the nuclear body in genomic stability. Oncogene, 18, 7941-7947. doi:10.1038/sj.onc. 1203367

[5] Reymond, A., Meroni, G., Fantozzi, A., Merla, G., Cairo, S., Luzi, L., Riganelli, D., Zanaria, E., Messali, S., Cainarca, S., Guffanti, A., Minucci, S., Pelicci, P. and Ballabio, A. (2001) The tripartite motif family identifies cell compartments. The EMBO Journal, 20, 2140-2151. doi:10.1093/emboj/20.9.2140

[6] Nenasheva, V., Maksimov, V., Nikolaev, A. and Tarantul, V. (2001) Comparative analysis of the level of gene transcription in two types of HIV-associated lymphoma. Mol Gen Mikrobiol Virusol, 4, 27-31.

[7] Reddy, B., Etkin, L. and Freemont, P. (1992) A novel zinc finger coiled-coil domain in a family of nuclear proteins. Trends in Biochemical Sciences, 17, 344-345. doi:10.1016/0968-0004(92)90308-V

[8] Borden, K. (1998) RING fingers and B-boxes: Zinc-binding protein-protein interaction domains. Biochemistry and Cell Biology, 76, 351-358. doi:10.1139/098-021

[9] Doetschman, T., Eistetter, H., Katz, M., Schmidt, W. and Kemler, R. (1985) The in vitro development of blastocyst-derived embryonic stem cell lines: Formation of visceral yolk sac, blood islands and myocardium. Journal of Embryology \& Experimental Morphology, 87, 27-45.

[10] Hübner, K., Fuhrmann, G., Christenson, L., Kehler, J., Reinbold, R., De La Fuente, R., Wood, J., Strauss, J., Boiani, M. and Schöler, H. (2003) Derivation of oocytes from mouse embryonic stem cells. Science, 300, 1251-1256. doi:10.1126/science. 1083452

[11] Wobus, A., Holzhausen, H., Jäkel, P. and Schöneich, J. (1984) Characterization of a pluripotent stem cell line derived from a mouse embryo. Experimental Cell Research, 152, 212-219. doi:10.1016/0014-4827(84)90246-5

[12] Geijsen, N., Horoschak, M., Kim, K., Gribnau, J., Egga, K. and Daley, G. (2004) Derivation of embryonic germ cells and male gametes from embryonic stem cells. $\mathrm{Na}$ ture, 427, 148-154.

[13] Bobrysheva, I., Grigorenco, A., Novosadova, E., Kal'ina, N., Arsenyeva, E., Grivennikov, I., Tarantul, V. and Ro- 
gaev, E. (2003) Effects of human presenilin 1 isoforms on proliferation and survival of rat pheochromocytoma cell line PC12. Biochemistry, 68, 611-617. doi:10.1023/A:1024605523743

[14] Laemmli, U. (1970) Cleavage of structural proteins during the assembly of the head of bacteriophage T4. Nature, 227, 680-685. doi:10.1038/227680a0

[15] Manuilova, E., Arsenyeva, N., Khaidarova, N., Shugurova, I., Tarantul, V. and Grivennikov, I. (2008) Different effects of regulatory genes (tat, nef) of human immunodeficiency virus type 1 (HIV-1) on the proliferation and differentiation of mouse embryonic stem cells in vitro. International Journal of Biomedical Science, 4, 29-37.

[16] Novosadova, E., Manuilova, E., Arsenyeva, E., Khaidarova, N., Dolotov, O., Inozemtseva, L., Kozachenkov,
K., Tarantul, V. and Grivennikov, I. (2005) Opposite effects increased and decreased expression of the pub gene on the formation embryoid bodies of embryonic stem cells in vitro. Cell Technologies in Biology and Medicine, 3, 174-179.

[17] Abujarour, R., Efe, J. and Ding, S. (2010) Genome-wide gain-of-function screen identifies novel regulators of pluripotency. Stem Cells, 28, 1487-1497. doi:10.1002/stem.472

[18] Novosadova, E., Manuilova, E., Arsenyeva, E., Lebedev, A., Khaydarova, N., Tarantul, V. and Grivennikov, I. (2009) Influence of $p u b$ gene expression on differentiation of mouse embryonic stem cells into derivatives of ecto-, meso-, and endoderm in vitro. Acta Naturae, 2, 93-97. 